'Departamento de Hematología y Oncología, Facultad de Medicina Pontificia Universidad Católica de Chile. Red de Salud UC-Christus. Santiago, Chile.

Trabajo no recibió financiamiento. Los autores declaran no tener conflictos de interés.

Recibido el 15 de junio de 2020, aceptado el 6 de agosto de 2020 .

Correspondencia a:

Dr. Mauricio Sarmiento Lira 85, 4to piso, Laboratorio de Hematología. Santiago, Chile. msarmiento@med.puc.cl

\section{Perspectiva hematológica en el tratamiento de pacientes con COVID-19: rol de los tratamientos antiinflamatorios}

\author{
PATRICIO ROJAS ${ }^{1}$, MAURICIO SARMIENTO ${ }^{1}$
}

\section{Anti-inflammatory therapies for SARS-COV2 infection from a hematological perspective}

The pathophysiology of the inflammatory profile induced by SARS-COV2 infection has similarities with conditions of immune system activation with cytokine release such as hemophagocytic syndrome and some cases of acute graftversus-host disease. There are encouraging results of clinical studies, performed with increasingly better methodological quality, supporting the use of targeted and specific anti-inflammatory therapy in selected groups of patients with $\mathrm{CO}$ VID-19 with severe inflammation. In this review we describe the inflammatory pathophysiology of the disease and the recent findings about its treatment.

(Rev Med Chile 2020; 148: 1640-1646)

Key words: COVID-19; Inflammation; Therapeutics.

\section{A} ctualmente la pandemia por COVID-19 tiene en una grave situación a la mayoría de los sistemas sanitarios del planeta con elevada contagiosidad y morbimortalidad. Este grave escenario e un problema de gran magnitud para los profesionales de salud y población general en cuanto a la calidad de la evidencia disponible que fue inicialmente muy deficiente y con una avalancha de información científica y de pseudociencia sobre tratamientos en algunos casos sin mayor fundamento fisiopatológico y sin el rigor científico requerido. Un claro ejemplo de esto se dio con el uso de hidroxicloroquina y azitromicina, en el que un trabajo de baja potencia, no revisado por pares y con dilemas éticos tuvo una expedita publicación y gran impacto mediático ${ }^{1}$, que sin embargo, al intentar reproducir los resultados con estudios de mejor calidad metodológica, no se obtuvieron resultados concordantes e incluso se describió en algunos casos efectos perjudiciales ${ }^{2}$. En el mismo sentido hay reportes con bajo número de pacientes que muestran experiencias con fármacos tales como tocilizumab, corticoides a dosis altas, plas- ma de convalecientes, interferón, incluso células mesenquimales, entre otros, que dada la discreta calidad metodológica hacen difícil incorporar potenciales terapias efectivas al arsenal clínico. Si bien la mayoría de los pacientes infectados por SARS-CoV-2 tienen un curso benévolo y con resolución espontánea, hasta un 5\% tendrán un cuadro clínico grave con falla orgánica pulmonar inicialmente, y otros un cuadro inflamatorio sistémico severo, que con tratamiento insuficiente puede desencadenar la muerte ${ }^{3}$.

Ahora bien, para muchos de los profesionales relacionados con la hematología, reumatología e inmunología, es muy llamativo el parecido de las manifestaciones graves de los pacientes con COVID-19 a lo que se observa en cuadros de síndrome hemofagocítico o de enfermedades inmunológicas graves. El objetivo de este manuscrito es mostrar nuestra visión como hematólogos con respecto a los fundamentos fisiopatológicos de enfermedades inflamatorias hematológicas graves y su correlación con la infección grave por SARS-CoV-2. 


\section{Fundamentos fisiopatológicos de infección grave por COVID-19}

Al analizar la fisiopatología la enfermedad grave por SARS-CoV-2, se ha reportado que en estos pacientes se desarrolla un proceso de activación linfocitaria muy intenso manifestado por el aumento en la producción y secreción de citoquinas IL-18, MCO1 (monocyte chemoattractant protein-1), IP10 (interferon gamma-induced protein 10) y MIP1a (macrophage inflammatory protein) como primera respuesta inmune, y como consecuencia, reclutamiento de linfocitos, macrófagos y polimorfonucleares, que a su vez secretan IL-15, IFNa/IFNb, IL-12 e IL-21 necesarias para el control de la infección viral. Lamentablemente la activación linfocitaria también promueve la secreción IL-6, TNF, IL-17a, GM-CSF, G-CSF que pueden inducir hiper inflamación sistémica y pulmonar ${ }^{4}$. Si este fenómeno hiper inflamatorio es persistente puede llevar a la falla respiratoria y en casos extremos a la falla multiorgánica. Además, con la liberación masiva de citoquinas (tormenta citoquínica) se ha descrito que los pacientes muestran niveles elevados de marcadores de activación macrofágica/síndrome hemofagocítico tales como ferritina, CD163 y CD25s (IL-2RA).

\section{Inhibición vía JAK/STAT e IL-6}

Los procesos de activación linfocitaria tienen diferentes vías susceptibles de ser inhibidas con tratamiento farmacológico. En los últimos años se ha descrito que la vía de JAK/STAT tiene un rol fundamental en diversas condiciones de inflamación excesiva como se observa en la enfermedad de injerto contra huésped (EICH), artritis reumatoide, colitis ulcerosa, psoriasis y síndrome hemofagocitico ${ }^{5,6}$. En contexto de la pandemia por COVID hay reportes que muestran eficiencia de Ruxolitinib, un inhibidor oral de las kinasas JAK1/ JAK2, lo que ha motivado que existan actualmente registrados en clinicaltrials.gov varios estudios en curso, algunos de ellos como estudio en Fase 3.

La razón para plantear el uso de inhibidores de la vía del JAK/STAT en la hiperactivación linfocitaria se basa en que esta vía de señalización tiene un rol indispensable en el proceso inflamatorio, dado que es necesaria tanto para la secreción como para la acción de diversas interleuquinas.
La fosforilación de las proteínas de la vía JAK es de alta eficiencia y de gran rapidez y hace que en menos de 30 min la mayoría de las vías de señalización intracelular se encuentren fosforiladas con la consecuente activación nuclear de factores de transcripción y producción de proteínas necesarias para la función del linfocito ${ }^{7}$. En los pacientes fallecidos por COVID-19 con falla respiratoria grave, se ha visto en necropsias una intensa inflamación linfocitaria a nivel alveolar, en conjunto con infiltración macrofágica tisular y microhemorragia ${ }^{8}$.

La posibilidad de inhibir la vía JAK/STAT puede permitir detener de manera rápida la activación y reclutamiento linfocitario inflamatorio. La utilización de Ruxolitinib disminuiría la secreción y actividad de IL-6, IL-13, MCP-1 y TNF- $\alpha$. Este efecto se ha demostrado en el tratamiento con terapia celular tipo CART-cell, donde el uso de Ruxolitinib previene el síndrome de liberación de citoquinas, sin disminuir la efectividad de la terapia celular ${ }^{10} \mathrm{y}$ además en casos de síndrome hemofagocítico refractario, donde su uso ha permitido detener la inflamación con buenos resultados ${ }^{11}$. Una ventaja de emplear esta inhibición es que es poco probable la ocurrencia de efectos adversos graves, debido a que se plantea utilizar una dosis baja por un período de tiempo muy acotado. Por lo tanto sería poco probable la aparición de citopenias significativas, y si bien está descrito con su aplicación un leve aumento de reactivaciones de tuberculosis y por virus de la familia Herpesviridae y hepatitis $\mathrm{B}^{12}$, esta situación se ha observado en contexto de tratamiento prolongado (de varios meses) y con dosis mayores principalmente en síndromes mieloproliferativos, no tanto así con dosis empleadas en tratamiento para cuadros inflamatorios, como EICH Aguda, síndrome hemofagocítico refractario y lo propuesto para COVID-19. Por otro lado, también hay información sobre que Ruxolitinib inhibiría la replicación viral del $\mathrm{VIH}^{13}$, y si bien, evidentemente no hay información precisa en relación a su uso en COVID-19, sus efectos antiinflamatorios sí podrían tener implicancias en la evolución de esta enfermedad ${ }^{14}$.

En el mismo sentido de inflamación descontrolada, está demostrado también que los pacientes con enfermedad grave se benefician del uso de Tocilizumab, un anticuerpo anti receptor de IL-6. Existe bastante experiencia del uso de este anticuerpo en Síndrome de Liberación de 
Citoquinas (Cytokine Release Syndrome, CRS) asociado a terapia celular tipo CART-cell, siendo tocilizumab considerado como el tratamiento de primera línea en esta situación ${ }^{15}$. En casos de CRS se ha descrito que mientras más elevados se encuentren los niveles de IL-6 y Proteína C Reactiva, más probabilidad existe de cuadros más severos, lo que ha hecho que el uso de tocilizumab en el uso de CART-cells sea protocolizado ${ }^{16,17}$. En infección grave por COVID-19 varios centros ya lo han utilizado en contexto de tormenta citoquínica, y las pocas series publicadas sus resultados serían auspiciosos en esta condición ${ }^{18}$. Es bien sabido que Italia fue duramente golpeado por la infección por SARS-CoV-2, por lo que guías locales han incluido tocilizumab $(8 \mathrm{mg} / \mathrm{Kg}$, con una segunda dosis $12 \mathrm{~h}$ post y una posible tercera post 24-36 h, según respuesta clínica) en caso de deterioro clínico y/o radiológico precoz ${ }^{19}$.

\section{Activación Macrofágica/Síndrome hemofagocitico Like}

Del punto de vista hematológico al revisar los cambios inmunológicos que induce el COVID-19, se puede observar que son muy similares a los cambios inducidos por el síndrome hemofagocítico. Esta grave enfermedad hematológica se clasifica como primaria o secundaria; en el tipo primario se da por déficit congénito de perforinas y reguladores de actividad macrofágica, y en la secundaria por diversas condiciones dentro de las que se incluyen neoplasias, infecciones virales y enfermedades autoinmunes ${ }^{20}$. En el síndrome hemofagocítico secundario se da una intensa tormenta citoquínica en contexto de una interacción exagerada y descontrolada de macrófagos y linfocitos T CD8. En este escenario se observa que la activación celular promueve elevados niveles de interleucinas inflamatorias IL-1, 6, 12, 15, 18, etc. Además se observa en estos casos una intensa hiperferritinemia asociada a una elevada producción de CD163, proteína tipo scavenger que es afín a la captación de proteínas heme y haptoglobina.

En modelos de infección por SARS-CoV en primates se ha visto que anticuerpos tipo IgG Anti-spike inducen cambios de fenotipo en macrófagos pulmonares de "reparadores" a "pro-inflamatorios" lo que generaría injuria pulmonar severa durante la infección aguda ${ }^{21}$.

En este sentido la tormenta citoquínica que induce la infección por COVID-19 es bastante similar a lo observado en el sindrome hemofagocitico de otras etiologías (Figura 1). Por lo tanto se ha planteado de forma cada vez más consistente por diversos autores que la estrategia antiviral en los casos hiper-inflamatorios, debe

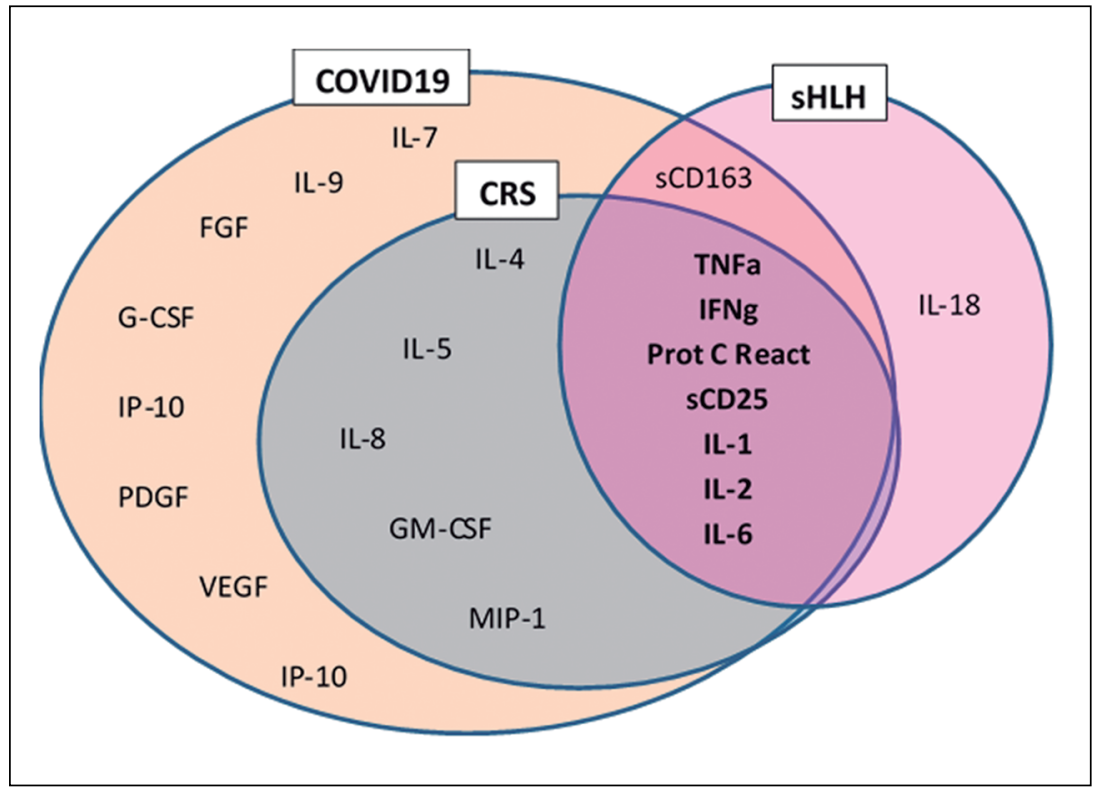

Figura 1. Comparación del perfil de citoquinas entre el $\mathrm{Sd}$ hemofagocitico secundario (sHLH), Sd de Liberación de Citoquinas (CRS) y el síndrome hiper inflamatorio por COVID-19. (Adaptado de Cancio M et al. ${ }^{28}$ ). 
ir acompañada de tratamiento dirigido a la tormenta citoquínica ${ }^{22,23}$.

En cuanto a las alteraciones de la coagulación se ha descrito que en los síndromes hemofagocíticos el fenómeno inflamatorio excesivo favorece activación endotelial, formación de microtrombos por generación de trombina y activación descontrolada de plasmina, con la consecuente prolongación de todos los tiempos de coagulación, consumo de fibrinógeno y elevación de dímero D. En los pacientes con infección grave por SARS-CoV-2, también se pueden encontrar fenómenos inflamatorios de la coagulación destacando la intensa elevación del dímero $\mathrm{D}$ y del fibrinógeno en la fase inicial de la infección; sin embargo en algunos series se ha observado que el descenso brusco del fibrinongeno se asocia con mal pronóstico ${ }^{24-26}$.

\section{Evidencia clínica disponible}

Como hemos descrito hay abundante sustrato fisiopatológico que muestra la similar actividad inflamatoria en los síndromes de activación macrofágica y en enfermedades inflamatorias tales como enfermedad de injerto vs huésped, con la inflamación inducida por la infección por COVID-19. En el inicio de la pandemia surgió variada información clínica, fundamentada en reportes de casos aislados o experiencias unicéntricas que hicieron difícil la interpretación de los hallazgos y más aun, su validación. Por fortuna últimamente han aparecido trabajos con adecuada calidad metodológica que nos pueden orientar un poco más sobre el rol de los tratamientos anti inflamatorios en pacientes infectados por COVID-19.

Uno de los primeros estudios publicados comparó ruxolitinib versus el mejor tratamiento disponible en pacientes con COVID-19 grave $^{30}$, en un estudio randomizado, prospectivo y multicéntrico realizado en China. Cuarenta y tres pacientes fueron aleatorizados para recibir ruxolitinib $5 \mathrm{mg}$ dos veces al día o placebo. Se incluyeron pacientes con COVID-19 "grave" (definido como hipoxemia $\mathrm{FiO}_{2}$ ambiental o frecuencia respiratoria $>30$ respiraciones/minuto), no intubado en el momento del reclutamiento, no embarazo o lactancia, y sin otra infección activa. La intervención se asignó en promedio día 20 de la infección, y el $70 \%$ en ambas ramas utilizó corticoides. Cuatro pacientes en el grupo de control se deterioraron clínicamente, mientras que ninguno de los pacientes en el grupo de ruxolitinib lo hizo ( $\mathrm{p}=$ $0,1)$. Tres pacientes en la rama placebo requirieron intubación y posteriormente fallecieron (14\%), mientras que ninguno falleció en el grupo de ruxolitinib (0\%) $(\mathrm{p}=0,232)$. Los pacientes en el grupo de ruxolitinib tuvieron mayor proporción de mejoría en escáner pulmonar en día 14 antes que la rama placebo $(\mathrm{p}=0,05)$.

En contexto de tocilizumab, fue publicado en un servicio "preprint" un estudio fase 2 que utilizó este tratamiento anti interleuquina 6 en 1.120 pacientes italianos, en que se demostró en comparación con grupo de control histórico, una reducción en mortalidad de 22,4 a $18,4 \%$ (97,5 IC: 14,2-28,3, p < 0,001). Este estudio sin embargo tiene diversas limitaciones metodológicas dentro de las que se incluyen, intervenciones concomitantes no controladas tales como uso de hidroxicloroquina, corticoides y $40 \%$ en ambas cohortes no usó el tocilizumab por lo que en el análisis por intención de tratar (no por protocolo) pudo haberse seleccionado la intervención a los pacientes más graves ${ }^{31}$. Otras experiencias en grupos pequeños de pacientes muestran rápido control de la fiebre, reducción de requerimientos de oxígeno y alta precoz ${ }^{32}$.

Distintos medicamentos hematológicos inhibidores de diversas vías inflamatorias se están probando en distintas fases de investigación y sin tener resultados aplicables a la práctica clínica aún (Tabla 1).

\section{Conclusiones}

La infección por SARS-CoV-2 promueve un grave proceso inflamatorio que puede ser letal. $\mathrm{La}$ interrupción del proceso inflamatorio viralmente inducido ha demostrado ser efectiva en casos con síndrome hiper inflamatorio, evitando el temido deterioro pulmonar progresivo y requerimientos de UCI y ventilación mecánica. En nuestro criterio, protocolizar el uso de inhibidores de JAK2 y anti interleuquina 6 es prioritario para los centros asistenciales de nuestro país, con el objetivo primordial de evitar la progresión inflamatoria grave y la necesidad de ventilación mecánica invasiva. El ruxolitinib y tocilizumab tienen evidencia creciente que muestra utilidad en este escenario 
Tabla 1. Algunos agentes terapéuticos hematológicos anti inflamatorios en estudio para el tratamiento de infección SARS-CoV-2

\begin{tabular}{|llc|}
\hline Agente & Usos habituales & $\begin{array}{c}\text { Fase de estudio en } \\
\text { COVID-19 }\end{array}$ \\
$\begin{array}{l}\text { Anticuerpos monoclonales } \\
\text { Tocilizumab } \\
\text { Begelomab }\end{array}$ & Artritis reumatoide, tormenta citoquínica, enf. Autoinmunes & Fase $2^{33}$ \\
$\begin{array}{l}\text { Inhibidores de BTK } \\
\text { Ibutinib/acalubrutinib }\end{array}$ & GVHD & Preclínico \\
$\begin{array}{l}\text { Inhibidores de JAK2 } \\
\text { Ruxolitinib } \\
\text { Baricitinib }\end{array}$ & Mielofibrosis, GVHD, & Fase $2^{34}$ \\
$\begin{array}{l}\text { Inhibidores tirosinkinasa } \\
\text { Nilotinib }\end{array}$ & GVHD, atopia & Fase $3^{30}$ \\
Dasatinib & Leucemia mieloide crónica & Preclínico \\
\hline $\begin{array}{l}\text { Interferones } \\
\text { GIST, GVHD }\end{array}$ & Preclínico \\
\hline
\end{tabular}

GVHD: graft versus host disease; SLP: síndromes linfoproliferativos; GIST: gastrointestinal stromal tumour; NMPCr: neoplasia mieloproliferativa crónica.

pero que dado el costo y riesgos asociados, debe hacerse una adecuada selección de los pacientes que realmente puedan beneficiarse del tratamiento. Una vez más, el trabajo multidisciplinario con concurso de infectólogos, urgenciólogos, internistas hospitalistas, broncopulmonares, intensivistas, reumatólogos y hematólogos, permitirán enfrentar de forma eficiente esta gran amenaza sanitaria.

\section{Referencias}

1. Gautret P, Lagier JC, Parola P, Hoang VT, Meddeb L, Mailhe M, et al. Hydroxychloroquine and Azithromycin as a Treatment of COVID-19: Results of an Open-Label Non-Randomized Clinical Trial. Int J Antimicrob Agents 2020.

2. Wei Tang, Zhujun Cao, Mingfeng Han, Zhengyan Wang, Junwen Chen, Wenjin Sun et al. Hydroxychloroquine in patients with COVID-19: an open-label, randomized, controlled trial. MedRxiv https://doi.org/ 10.1101/2020.04.10.20060558).

3. Ahn DG, Shin HJ, Kim MH, Lee S, Kim HS, Myoung J, et al. Current Status of Epidemiology, Diagnosis, Therapeutics, and Vaccines for Novel Coronavirus Disease 2019 (COVID-19). J Microbiol Biotechnol 2020; 30 (3): 313-24. doi: 10.4014/jmb.2003.03011.
4. Schett G, Sticherling M, Neurath MF. COVID-19: risk for cytokine targeting in chronic inflammatory diseases? Nat Rev Immunol 2020; 20 (5): 271-2. doi: 10.1038/ s41577-020-0312-7.

5. Birndt S, Schenk T, Heinevetter B, Brunkhorst FM, Maschmeyer G, et al Hemophagocytic lymphohistiocytosis in adults: collaborative analysis of 137 cases of a nationwide German registry. J Cancer Res Clin Oncol 2020; 146 (4): 1065-77.

6. Sarmiento Maldonado M, Ramírez Villanueva P, Bertín Cortes-Monroy P, Jara Arias V, Soto Donoso K, Uribe González P, et al. Compassionate use of ruxolitinib in acute and chronic graft versus host disease refractory both to corticosteroids and extracorporeal photopheresis. Exp Hematol Oncol 2017; 6: 32.

7. Duarte R, Frank D. La vía JAK-STAT de señalización intracelular y su repercusión en oncogénesis, inmunomodulación y desarrollo. Medicina Clínica 2000; 114 (6): 227-34.

8. Hanley B, Lucas SB, Youd E, Swift B, Osborn M. Autopsy in suspected COVID-19 cases. J Clin Pathol 2020; 73 (5): 239-42. doi:10.1136/jclinpath-2020-206522.

9. Hermans MAW, Schrijver B, van Holten-Neelen CCPA, Gerth van Wijk R, van Hagen PM, et al. The JAK1/ JAK2- inhibitor ruxolitinib inhibits mast cell degranulation and cytokine release. Clin Exp Allergy 2018; 48 (11): 1412-20. 
10. Khadka RH, Sakemura R, Kenderian SS, Johnson AJ.. Management of cytokine release syndrome: an update on emerging antigen-specific $\mathrm{T}$ cell engaging immunotherapies. Immunotherapy 2019; 11 (10): 851-7.

11. Broglie L, Pommert L, Rao S, Thakar M, Phelan R, Margolis D, et al. Ruxolitinib for treatment of refractory hemophagocytic lymphohistiocytosis. Blood Adv 2017; 1 (19): 1533-6.

12. Elli EM, Baratè $\mathrm{C}$, Mendicino $\mathrm{F}$, Palandri $\mathrm{F}$, Palumbo GA. Mechanisms Underlying the Anti-inflammatory and Immunosuppressive Activity of Ruxolitinib. Front Oncol 2019; 9: 1186. Published 2019 Nov 7. doi:10.3389/ fonc.2019.011861.

13. Gavegnano C, Detorio M, Montero C, Bosque A, Planelles V, Schinazi RF. Ruxolitinib and tofacitinib are potent and selective inhibitors of HIV-1 replication and virus reactivation in vitro. Antimicrob Agents Chemother 2014; 58 (4): 1977-86. doi:10.1128/AAC.02496-13.

14. Tuttle K, Minter R, Waugh K, Araya P, Ludwig M, Sempeck C, et al. JAK1 inhibition blocks lethal sterile immune responses: implications for COVID-19 therapy. bioRxiv 2020.04.07.024455; doi: https://doi. org/10.1101/2020.04.07.024455.

15. Kotch C, Barrett D, Teachey DT. Tocilizumab for the treatment of chimeric antigen receptor $\mathrm{T}$ cell-induced cytokine release syndrome. Expert Rev Clin Immunol 2019; 15 (8): 813-22.

16. Porter DL, Hwang WT, Frey NV, Lacey SF, Shaw PA, Loren AW, et al. Chimeric antigen receptor $\mathrm{T}$ cells persist and induce sustained remissions in relapsed refractory chronic lymphocytic leukemia. Sci Transl Med 2015; 7 (303): 303ra139.

17. Maude SL, Frey N, Shaw PA, Aplenc R, Barrett DM, Bunin NJ, et al. Chimeric antigen receptor $\mathrm{T}$ cells for sustained remissions in leukemia. N Engl J Med 2014; 371 (16): 1507-17.

18. Luo P, Liu Y, Qiu L, Liu X, Liu D, Li Jl. Tocilizumab treatment in COVID-19: A single center experience. J Med Virol 2020; 10.1002/jmv.25801. doi:10.1002/ jmv.25801.

19. Di Giambenedetto S, Ciccullo A, Borghetti A, Gambassi G, Landi F, Visconti E, et al. Off-label Use of Tocilizumab in Patients with SARS-CoV-2 Infection. J Med Virol 2020; 10.1002/jmv.25897.

20. Schulert GS, Grom AA. Pathogenesis of Macrophage Activation Syndrome and Potential for Cytokine- Directed Therapies. Annu Rev Med 2015; 66: 145-59. doi: .1146/ annurev-med-061813-012806. Epub 2014 Nov 5).

21. Liu L, Wei Q, Lin Q, Fang J, Wang H, Kwok H, et al. Anti-spike IgG causes severe acute lung injury by skewing macrophage responses during acute SARS-CoV infection. JCI Insight 2019; 4 (4): e123158. Published 2019 Feb 21. doi: 10.1172/jci.insight.123158.

22. Stebbing J, Phelan A, Griffin I, Tucker C, Oechsle O, Smith D, et al. COVID-19: Combining Antiviral and Anti-Inflammatory Treatments. Lancet Infect Dis 2020; 20 (4): 400-2. doi: 10.1016/S1473-3099(20)30132-8. Epub 2020 Feb 27.

23. Favalli EG, Ingegnoli F, De Lucia O, Cincinelli G, Cimaz R, Caporali R. COVID-19 infection and rheumatoid arthritis: Faraway, so close! Autoimmun Rev 2020; 102523. doi: 10.1016/j.autrev.2020.102523 [Epub ahead of print].

24. Valade S, Mariotte E, Azoulay E. Coagulation Disorders in Hemophagocytic Lymphohistiocytosis/Macrophage Activation Syndrome. Crit Care Clin 2020; 36 (2): 41526. doi:10.1016/j.ccc.2019.12.004.

25. Lee N, Hui D, Wu A, Chan P, Cameron P, Joynt GM, et al. A Major Outbreak of Severe Acute Respiratory Syndrome in Hong Kong. N Engl J Med 2003; 348: 1986-994.

26. Connors JM, Levy JH. COVID-19 and its implications for thrombosis and anticoagulation. Blood 2020; 135 (23): 2033-40. doi: 10.1182/blood.2020006000. PMID: 32339221; PMCID: PMC7273827.

27. Porter D, Frey N, Wood PA, Weng Y, Grupp SA. Grading of cytokine release syndrome associated with the CAR T cell therapy tisagenlecleucel. J Hematol Oncol 2018; 11 (1): 35. doi: 10.1186/s13045-018-0571-y. Erratum in: J Hematol Oncol. 2018; 11 (1): 81. PMID: 29499750; PMCID: PMC5833070.

28. Lee DW, Santomasso BD, Locke FL, Ghobadi A, Turtle CJ, Brudno JN, et al. ASTCT Consensus Grading for Cytokine Release Syndrome and Neurologic Toxicity Associated with Immune Effector Cells. Biol Blood Marrow Transplant 2019; 25 (4): 625-38.

29. Cancio M, Ciccocioppo R, Rocco PRM, Levine BL, Bronte V, Bollard CM, et al. Emerging Trends in COVID-19 Treatment: Learning from Inflammatory Conditions Associated with Cellular Therapies. Cytotherapy 2020. doi: 10.1016/j.jcyt.2020.04.100.

30. Cao Y, Wei J, Zou L, Jiang T, Wang G, Chen L, et al. Ruxolitinib in treatment of severe coronavirus disease 2019 (COVID-19): A multicenter, single-blind, randomized controlled trial. J Allergy Clin Immunol 2020; S00916749(20)30738-7. doi:10.1016/j.jaci.2020.05.019.

31. Perrone F, Piccirillo MC, Ascierto P, Salvarani C, Parrella $\mathrm{P}$, Marata A, et al. Tocilizumab for patients with COVID-19 pneumonia. The TOCIVID-19 phase 2 trial. medRxiv 2020.06.01.20119149; doi: https://doi.org/10.1 101/2020.06.01.20119149.

32. Xu X, Han M, Li T, Sun W, Wang D, Fu B, et al. Effecti- 
ve treatment of severe COVID-19 patients with tocilizumab. Proc Natl Acad Sci U S A. 2020;117(20):10970-5. doi:10.1073/pnas.2005615117.

33. Maes B, Bosteels C, De Leeuw E, Declercq J, Van Damme K, Delporte A, et al. Treatment of severely ill COVID-19 patients with anti-interleukin drugs (COVAID): A structured summary of a study protocol for a randomised controlled trial. Trials 2020; 21 (1): 468. Published 2020 Jun 3. doi:10.1186/s13063-020-04453-5.

34. Treon SP, Castillo JJ, Skarbnik AP, Soumerai JD, Ghobrial IM, et al. The BTK inhibitor ibrutinib may protect against pulmonary injury in COVID-19-infected patients. Blood 2020; 135 (21): 1912-5. doi:10.1182/ blood.2020006288.

35. Cantini F, Niccoli L, Matarrese D, Nicastri E, Stobbione P, Goletti D. Baricitinib therapy in COVID-19: A pilot study on safety and clinical impact [published online ahead of print, 2020 Apr 23]. J Infect 2020; S01634453(20)30228-0. doi:10.1016/j.jinf.2020.04.017.

36. Dyall J, Coleman CM, Hart BJ, Venkataraman T, Holbrook MR, Kindrachuk J, et al. Repurposing of clinically developed drugs for treatment of Middle East respiratory syndrome coronavirus infection. Antimicrob Agents Chemother 2014; 58 (8): 4885-93. doi: 10.1128/ AAC.03036-14. Epub 2014 May 19. PMID: 24841273; PMCID: PMC4136000.

37. Hung IF, Lung KC, Tso EY, Liu R, Chung TW, Chu MY, et al. Triple combination of interferon beta- $1 \mathrm{~b}$, lopinavir-ritonavir, and ribavirin in the treatment of patients admitted to hospital with COVID-19: an open-label, randomised, phase 2 trial. Lancet 2020; 395 (10238): 1695-704. doi: 10.1016/S0140-6736(20)31042-4. 